\title{
Analysis of Racial Discrimination in Artificial Intelligence from the Perspective of Social Media, Search Engines, and Future Crime Prediction Systems
}

\author{
Xinxun $\mathrm{Li}^{1, *}$
}

${ }^{1}$ Emory College, Emory University, Atlanta, GA 30322, USA

*Corresponding author. Email: lindaxinxun@gmail.com

\begin{abstract}
With the development of society in general, morality is also evolving. Some may argue that racial equality will be eventually achieved. However, this optimistic thought may be really hard to realize, especially under the development of artificial intelligence because AI (Artificial Intelligence) is invented by humans, and it is humans who code and input the codes into the artificial intelligence system. If anyone of the inventors or programmers is racially biased, the algorithms they code will also be racially discriminated against minorities. Based on the document study and summary, this paper will examine the aspects of AI causing racial discrimination, existing solutions and possible solutions. At the end of the study, this paper presents that although there are some laws and public policies that may be helpful to racial discrimination, they are not enough. The solutions should not only limit to the state level but also should include company and individual levels. Lastly, in order to overcome racial discrimination, only focusing on algorithms or AI itself is not enough. Discrimination comes from multiple aspects, and if society cannot dedicate to trying to solve all of them, the concept or the mindset of racial crimination can still have a negative impact on AI eventually.
\end{abstract}

Keywords: Artificial intelligence, Racial discrimination, Algorithms, Public policy.

\section{INTRODUCTION}

Nowadays with the development of Artificial Intelligence, people's life is becoming easier and easier than before. When people are using social media like Twitter and Facebook to search for news, information, articles that they are interested in, they do not have to type in every single topic that they are curious about. By learning individuals' personal information, AI can push different advertisements and recommendations to users based on their gender, race, interests, etc. This seems very appealing to society and people can imagine the algorithm itself can do the calculation and interpretation of personal information, and then customize recommendations. However, the questions are: whether $\mathrm{AI}$ exposes people to the information they actually want to know; what other information AI would use to make the calculation, whether AI does the measurement accurately, and whether, AI can distribute information justly and reasonably. Those problems are hard to address. Besides, nowadays there is no real Artificial Intelligence and all society has is intellectual automation, which means that no one can promise that algorithms themselves can interpret and judge the information on a fair scale. As a result, humans' value, especially programmers and companies', is the main factor in deciding how to make the decision and what measurement should be made for users. If there is bias, it is not the problem of the technology itself, but it is the problem of humans. Moreover, since the world is so diverse, it is hard to invent promising AI that can represent every culture or race's will. Therefore, discrimination can be easily found. The main goal of the first part of this paper will be examining racial discrimination in AI especially in advertisement and prediction of future crimes using past document study. Then the paper will present the existing solutions and some 
potential solutions that can better solve racial discrimination. This study is meaningful to human society as the world itself should be one diverse and just unity, rather than multiple groups with hostility and polarization.

\section{RACIAL DISCRIMINATION}

The first question that this paper will be discussing is the situation of racial discrimination. Multiple aspects show the problem of discrimination. However, this paper will examine two aspects in-depth, namely future crime and advertisement. This paper will interpret how social media, search engines, and future crime prediction systems discriminate different against races.

\subsection{Racial Discrimination in Police Data}

It is not a brand-new discovery that Artificial Intelligence has been used in the legal and judicial system. When it comes to dealing with crimes or legal issues, humans need an impartial judge, and in order to maintain security, future crime prediction is also very important. Therefore, society introduces $\mathrm{AI}$ to take on this responsibility and it seems practical to some people as AI has no preference to either side when it comes to issues about criminals. However, this sense of objectivity is the veil of lies. Just in late 2013, an African American named Robert McDaniel was warned not to commit future crimes [1]. The funny thing was that McDaniel had no record showing past crimes. The only reason for him being warmed was simply because of where he had lived - the South Side of Chicago. It turns out that the machine learning algorithm is biased as it only interprets geographic location and arrest data that are not being randomized nor being representative enough. As a result, the warning itself may be inaccurate to both neighborhood and those who are being warned.

Living in a community that used to or currently has a high rate of crime does not necessarily mean that an individual will commit a crime in the future. If the warning is announced in the public, it might cause unnecessary anxiety and panic to the community and also a sense of being affronted and distrusted by the individual who is being falsely warned. A high-crime-rate neighborhood has lawabiding citizens, and similarly, a none-crimereported county may still have criminals or potential criminals hiding. The factor that causes someone to $\sin$ is not where he or she lives, but the biology and living conditions. It is hard to measure every place and every race's rate of crime throughout the world. Therefore, it is necessary to make the police recording data and predicting system transparent to a certain degree so that the rest of the society would know its scale and how promising this prediction might be.

Additionally, what also causes unreliability of the prediction is that many of the reports were in fact citizen-reporting rather than police reports [1]. Citizen-reported crime can be mistaken and trustless. Firstly, because not all the citizens are trained professionally, they may predict incidents that are not a crime. Besides, humans are not always rational. Some people are highly cynical and tend to distrust others. As a result, they are more likely to report false predictions and suspicion. Lastly, according to "report suspicious activity" on the homeland security report website [2], reporters are required to provide descriptions of activities and physical identities according to their observations. Nonetheless, when someone is being anxious and panic because he or she sees something or someone suspicious, they may exaggerate the fact on the report. This also results in inaccuracy of the fact.

\subsection{Racial Discrimination in COMPAS Algorithm}

Other examples including Brisha Borden, an African American woman, after taking a kid's bike and scooter, she was being rated as high risk for future crime. Ironically, she did not re-offend later [3]. On the other hand, Vernon Prater, a white man, being rated as low risk, had one subsequent offense later [3]. The mistake of COMPAS algorithm raises attention, and it was found out that "Black defendants were still 77 percent more likely to be pegged as at higher risk of committing a future violent crime and 45 percent more likely to be predicted to commit a future crime of any kind" [3].

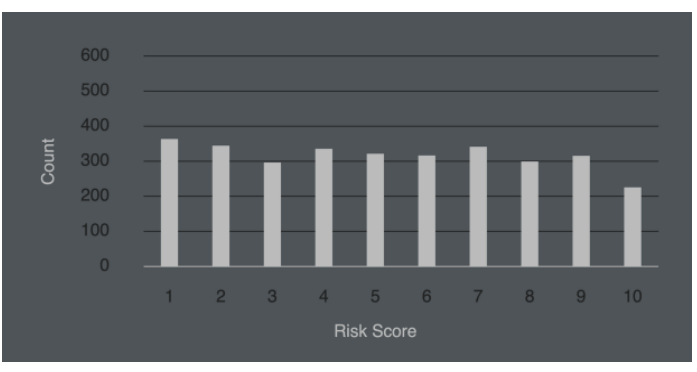

Figure 1 Black defendants' risk score [3]. 


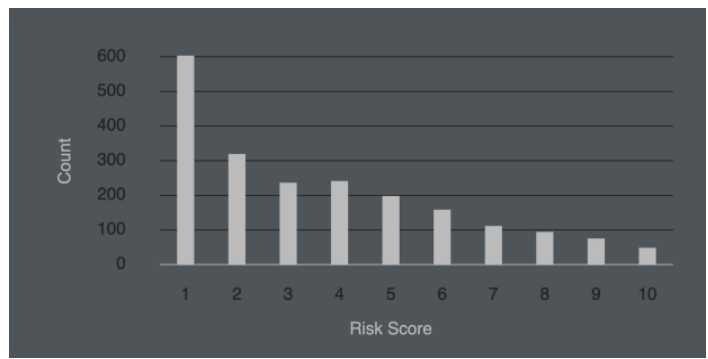

Figure 2 White defendants' risk score [3].

"Figure 1" and "Figure 2" together show that the prediction of African Americans' and white people's potential for offenses are largely different. White people are much less likely to re-offend than African Americans, but the reasons are not clear.

Therefore, it is important to find out why African Americans are more likely to be rated as high-risk. Once again, technologies themselves are not biased, but the design of algorithms by developers may be. If the training data are not being randomized, or more examples are African American offenders in the training data, this may cause false interpretation by algorithms such as COMPAS. Therefore, it is important to notice what type of crimes, offenses, and how many white offenders and black offenders are being collected. It is also crucial to pay attention to within those training data who commit what kind of crime because if all the felony are caused by African Americans, while most of the white men in the training data are only responsible for a misdemeanor, this can also cause racial bias when predicting future crime. What's more, the evenness and fairness of different races' backgrounds, past recordings are also important because Artificial Intelligence does not have cognition as humans do. Hence, they are not able to make the measurement independently and fairly - they need humans to provide a just scale.

But the obstacle that is preventing the improvement of COMPAS system is that the developers of the COMPAS software rejected disclosing information about their algorithms [4]. As a result, it is almost impossible to access the training data and overcome racial discrimination.

\subsection{Racial Discrimination in Political Advertising}

In a democratic country, as the master of the country, citizens usually attach importance to politics, especially political campaigns, and participate in it. However, because the identity of citizens instead of politicians, people's means of getting the news and information of campaigns largely depend on social media. One big problem with social media is reliability. It is vital to figure out whether the news is accurate or not, and whether reporters are reliable and neutral enough.

In 2016, the black voter turnout rate declined, for the first time within the twenty years because of Russian intervention during the campaign [5]. Russian targeting operative targeted African Americans by making African nationals post content about racial discrimination and racial segregation [6]. The contents were negative tweets about "white police", "African Americans" related content that can raise racial tension. Because of the limitations of algorithms, when there are African nationals intentionally making posts that relate to bias and division, algorithms would learn that people that may be African Americans need similar advertisements. Therefore, more and more African American users would be recommended with advertisements about inequality and discrimination. Although social media operators or companies do not intend to spread these types of advertisements, the algorithms cannot discern and make measurements of how necessary or important it is for users to see this advertisement.

Inadvertent bias in recommendations might cause dissatisfaction and turmoil. Furthermore, because of democracy, people are used to practicing their thoughts. As a result, the use of algorithms might not help people participate in politics but rather becomes the weapon utilized by others to violate or further intensify racial relationships.

\subsection{Racial Discrimination in the Job Advertisement}

In 2015, amazon found out that their recruiting engine was not functioning in a gender-neutral way. This is because the algorithm discovers that over the past ten years, the technology industry was almost dominated by males. As a result, the recruiting engine penalized resumes that mention words related to "woman" [7]. Although this discrimination happened in gender and nonadvertising area, it is very likely to happen in advertisements for different races one day. This is because from this example, we see the limit and bias of algorithms and they make decisions based on patterns. If algorithms learn that most African Americans are cleaners at a certain university, and when an African American wants to apply for a job 
in this university, the most possible advertisement that will be recommended to him or her is not being a professor, a treasurer, but a cleaner. This logic can be generalized to all kinds of job advertisements. This racial bias can violate diversity and equal opportunities. Being shielded from some jobs, African Americans are barely possible to consider them, and therefore, those jobs can be white-dominated. This is harmful to diversity as for example if all the professors are white people; it is hard for the school to have different voices and perspectives on things. Since humans are biocultural, which means they are shaped by both biology and culture, different culture and races create abundant thoughts. This is also a violation of equal opportunity as in a democratic society, equality, fairness, and justice are important. Hence, not giving people the equal opportunity to be exposed to the same job advertisements prevents people from having equal opportunities for different positions.

\section{EXITING SOLUTIONS}

Since there is so much racial bias in algorithms, it is important to actively seek solutions. Indeed, there are some legal protections. For instance, Title 42, Chapter 21 of the U.S. Code protects many different cultures and groups of people's rights from discrimination in various settings like education and employment [8]. Citizens and immigrants can better understand their rights and how to exercise them through help from a National Origin Working Group established by the Civil Rights Division [9]. Sometimes, it is hard for individuals to realize all of their rights, and people can be easily cheated when they are unclear about their legal rights and duties. Hence, it is important to establish organizations like this to provide people with legitimate authority to appeal to. Besides, taking housing as an example, there is the Fair Housing Act, which can protect different groups of people from discrimination [10]. Additionally, there is "FTC's law enforcement actions, studies, and guidance emphasize that the use of AI tools should be transparent, explainable, fair, and empirically sound while fostering accountability" [11]. Unfortunately, the law and public policies that directly address racial problems in AI are not enough. Similarly, the company level and individual level are not giving sufficient attention.

\section{POSSIBLE SOLUTIONS AND SUGGESTIONS}

\subsection{State}

It is always necessary for the state to intervene and set up fair policies. Therefore, the first issue that the government needs to address is making the process of collecting training data and algorithms transparent. Such as the first issue in this paper, which is the police data, the government is supposed to supervise citizen-reported cases, examining the accuracy and going a step further on what needs to be done when reporting a case, including objective details rather than subjective feelings. Besides, it is also important for the state to make sure that the database uses fair and even data of records among different races and locations. To be more specific, for instance, when selecting data about a felony, it is important to include as many locations as possible and also to include different races' number of crimes in a relatively similar number. Other than mere regulations and take specific steps toward a strict regulation, laws are also necessary. The state can make laws that set the standard of transparency that companies should meet for inventing, using, and selling AI. Moreover, the law also needs to enforce companies to publish and be honest to the public that what kind of database they use, how they collect training data, and the scale of machine learning uses to serve people.

In general, the state is supposed to have both the efficient and powerful force and regulations on transparency, social media regulations, and companies' means and ends. The first two are intuitive. The previous paragraph is about regulations on transparency. For social media regulation, the government can set up a specific group or department in charge of filtering and supervising content. As for the last one, the regulation on companies' means and ends, this is because the government should make sure that the way companies achieve their goals are just and their goal is also fair. Additionally, the state also needs to make sure that it practices laws and policies efficiently. This can be hard as this relates to having a righteous authority that works for the common good. The government officials also need to be responsible and never slack off because nowadays there are many situations that officials are not being patient or helpful enough. For example, after filing a police report about fraud, there can be no more feedback or follow-up. 
Therefore, laws and policies should not merely be a placeholder while doing nothing practically. Punishment and deterrence should be taken into consideration. If there are organizations, corporations, and individuals violating laws and ethics, the state should have effective penalties. This is similar to what John Locke would argue that when people enter the society, they yield their rights to punish to the state. As a result, the state should be an effective place for the public to appeal to. Besides, punishment should let the offenders regret and have the effect of deterrence.

Lastly, a very important but often overlooked strategy is education. Technology itself is not biased, at least for now, but humans are because of what they learned from their cultural background and both past and current environment. Human nature can be shaped by the environment so when developers are inventing algorithms, their values may be deeply rooted in their mindset. Therefore, they can make racially discriminated machine learning consciously or subconsciously. Subconsciousness is more dangerous since it is hard to realize. The way to solve it is to create a just society, where people can be raised in education and culture that practice and value racial equality. When talking about education, it is about all-sided education, and education in science, business, and history and so on all need to dedicate to justice so that people can stay away from injustice and be shielded from unfair values even better.

\subsection{Company}

At the company level, the prime responsibilities include obeying the law and public policies and being careful about candidates and applicants. To be more specific, when selecting employees, their values and personalities are important because those factors can largely impact the algorithms they develop. Therefore, training in business ethics is also very crucial. Making algorithms transparent is a disputable topic because transparency may violate privacy and confidentiality. However, companies should keep in mind that the common good is the priority of society. Therefore, they do not have to publish their algorithms to the entire world, but there should be governmental departments regulating and supervising algorithms, and companies have to present their actual algorithms to the third party so that the black box situation can be reduced. The solution of racial issues should consider the perspective of racial prejudice.
Training data bias, how algorithms are designed, and decisions driven by algorithms are reasons that AI produces bias. As a result, it is vital to collect fair and even training data so that the pattern that AI learns will reduce bias.

Furthermore, it is important to clarify the purpose of developing certain algorithms and the entire society should have the access to the purpose. This is because society needs to prevent illegal or unethical transactions. For instance, if A company wants B company to develop AI that can eliminate African American candidates, and B did so for high profits in exchange. As a result, racial discrimination will never be possible to be reduced or eliminated, but can even cause more intense racial polarization, which is not beneficial to develop unity. Moreover, users have the right to know what a certain AI is used for and what the possible results are. For example, when they first use Facebook, Facebook should send them request such as "Do you want to receive job recommendations?" but this is not enough, it should also give them a brief list about in general, what kind of people pursued what kind of jobs successfully, and what database and training data the companies use. The list does not need to show the actual algorithms since most people do not understand it anyways and it may divulge confidentiality, but it is necessary to show the public the pattern and reference companies use for machine learning. In this way, users can better consider if they want to accept the recommendation and use the product or not.

Finally, when developing algorithms, companies should tell AI what customers and users want from its function. Making accurate and precise goals and decisions can reduce confusion and error.

\subsection{Individual Level}

In order to protect their own liberty and rights, people should be proactive to fight for their good. People need to explore and read carefully about the law and policies because it is hard for people to understand or even realize their rights without doing so. Therefore, after studying more laws and policies, the public will become more sensitive at observing issues, if AI violates their rights and good.

Moreover, once companies start using questionnaires and share lists about their products, users need to read and think about the information 
carefully. This shows people's respect for companies and also being accountable to themselves.

\section{CONCLUSION}

To sum up, the elimination of racial bias is a very hard and long journey that requires every aspect of society to dedicate. Justice in AI will only be achieved through each person's contribution. In order to solve the problem, society needs to find out the reason and origin of bias. Thus, the state, the company, and the individuals know what to do. This is a very complicated and tricky process, and it requires patience and promising actions.

Last but not the least, in order to reduce racial discrimination, it is not enough to just focus on AI. Discrimination may be a habit, a thought, or even a social ideology that can be formed because of the influence from any circumstance, any events or person. It requires regulation of reducing racial discrimination in every aspect of our life.

\section{AUTHORS' CONTRIBUTIONS}

This paper is independently completed by Xinxun Li.

\section{ACKNOWLEDGMENTS}

Thanks for the help from Sonia Katyal, a law professor at the University of California, Berkeley.

\section{REFERENCES}

[1] Lum, Kristian and Isaac, William. To predict or serve? Royal Statistical Society, rss.onlinelibrary.wiley.com/doi/full/10.1111/j. 1740-9713.2016.00960.x. October 7, 2016.

[2] Report Suspicious Activity. Homeland Security, www.dhs.gov/how-do-i/reportsuspicious-activity.

[3] Angwin, Julia and Larson, Jeff and Mattu, Surya and Kirchner, Lauren. Machine Bias. Propublica,

www.propublica.org/article/machine-biasrisk-assessments-in-criminal-sentencing. May 23, 2016.

[4] Alex. Racial Bias and Gender Bias Examples in AI system. THE COMUZI JOURNAL, medium.com/thoughts-and-reflections/racialbias-and-gender-bias-examples-in-ai-systems7211e4c166a1. September 2, 2018.
[5] Harrison, Dominique. Civil Rights Violations in the face of Technological Change. Aspeninstitute, https://www.aspeninstitute.org/blogposts/civil-rights-violations-in-the-face-oftechnological-change/. OCTOBER 22, 2020

[6] Švedkauskas, Žilvinas, and Sirikupt, Chonlawitand and Salzer, Michel. Russia's disinformation campaigns are targeting African Americans. Washingtonpost, https://www.washingtonpost.com/politics/202 0/07/24/russias-disinformation-campaigns-aretargeting-african-americans/

[7] Harrer, Andrew. Amazon scraps a secret A.I. recruiting tool that showed bias against women. CNBC, www.cnbc.com/2018/10/10/amazon-scraps-asecret-ai-recruiting-tool-that-showed-biasagainst-women.html

[8] Civil right. United State Code, uscode.house.gov/view.xhtml?path=/prelim@t itle42/chapter21\&edition=prelim.

[9] FEDERAL PROTECTIONS AGAINST NATIONAL ORIGIN DISCRIMINATION, The United States Department of Justice, www.justice.gov/crt/federal-protectionsagainst-national-origin-discrimination-1

[10] Federal Trade Commission. Big data A Tool for Inclusion or Exclusion? FTC report, www.ftc.gov/system/files/documents/reports/b ig-data-tool-inclusion-or-exclusionunderstanding-issues/160106big-data-rpt.pdf. January 2016.

[11] Smith, Andrew Director, FTC Bureau of Consumer Protection. Using Artificial Intelligence and Algorithms. Federal Trade Commision,www.ftc.gov/newsevents/blogs/business-blog/2020/04/usingartificial-intelligence-algorithms. April 8, 2020 . 